ECS Transactions, 85 (8) 103-109 (2018)

10.1149/08508.0103ecst @The Electrochemical Society

\title{
Parasitic Conduction on $\Omega$-Gate Nanowires SOI nMOSFETs
}

\author{
V.C.P. Silva ${ }^{\text {a }}$ J.A. Martino ${ }^{\mathrm{a}}$ and P.G.D. Agopian ${ }^{\mathrm{a}, \mathrm{b}}$

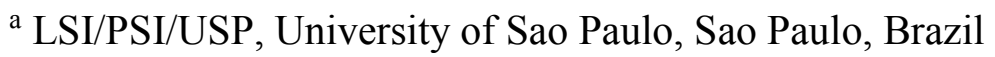 \\ ${ }^{\mathrm{b}}$ Sao Paulo State University (UNESP), Sao Joao da Boa Vista, Brazil \\ correspondent author email: vcpsilva@usp.br
}

In this work is analyzed the influence of fixed charges and interface traps on the subthreshold region of $\Omega$-Gate Nanowires SOI nMOSFETs devices with different channel width $\left(\mathrm{W}_{\text {fin }}\right)$ and length (L). The implementation of the fixed charges on the back interface (channel/buried oxide) results in a parasitic conduction in this region, reducing the effective threshold voltage of the transistor. It was verified that the presence of these fixed charges and of the interface traps hardly affect the subthreshold swing due to the strong coupling among the interfaces.

\section{Introduction}

The use of low-power low-voltage (LPLV) devices becomes even more important nowadays where microelectronics products are present on everyone's life, as medical equipment (hearing aids and pacemaker), smart watches, self-powered devices and microsensors, as habitat monitoring, health, structural monitoring, and automotive sensing (1)(2) . The circuits operating on the subthreshold region are used on applications where the reduction of power consumptions is more important than the performance. Since the subthreshold current is exponentially related to the gate voltage, this relationship is expected to give an exponential reduction in power consumption, making operating in this region advantageous for the applications mentioned before (2).

The $\Omega$-Gate nanowire (NW) SOI nMOSFETs is an excellent candidate for future technology nodes. The goal of this work is to study the $\Omega$-Gate NW SOI nMOSFET working in subthreshold region for low-power low-voltage (LPLV) application, with special attention on a possible parasitic drain current conduction.

\section{Devices Characteristics}

The studied devices are $\Omega$-Gate nanowires SOI nMOSFETs that were fabricated at CEA-LETI/France with the following characteristics: the effective oxide thickness (EOT) of $1.3 \mathrm{~nm}$, the buried oxide thickness $\left(\mathrm{t}_{\mathrm{oxb}}\right)$ of $145 \mathrm{~nm}$ and the channel height $\left(\mathrm{h}_{\text {fin }}\right)$ of $11 \mathrm{~nm}$ with three channel width ( $\mathrm{W}_{\mathrm{NW}}$ ) 220, 40 and 10nm and four channel lengths (L) 40, 100, $200 \mathrm{~nm}$ and $1 \mu \mathrm{m}$. In this work, the channel width $\left(\mathrm{W}_{\mathrm{NW}}\right)$ refers to the horizontal diameter of the transistor channel.

Figure 1 shows scanning electron microscopy (SEM) (left) and cross-sectional transmission electron microscopy (TEM) (right) images of $\mathrm{t} \Omega$-Gate NW SOI MOSFETs. 

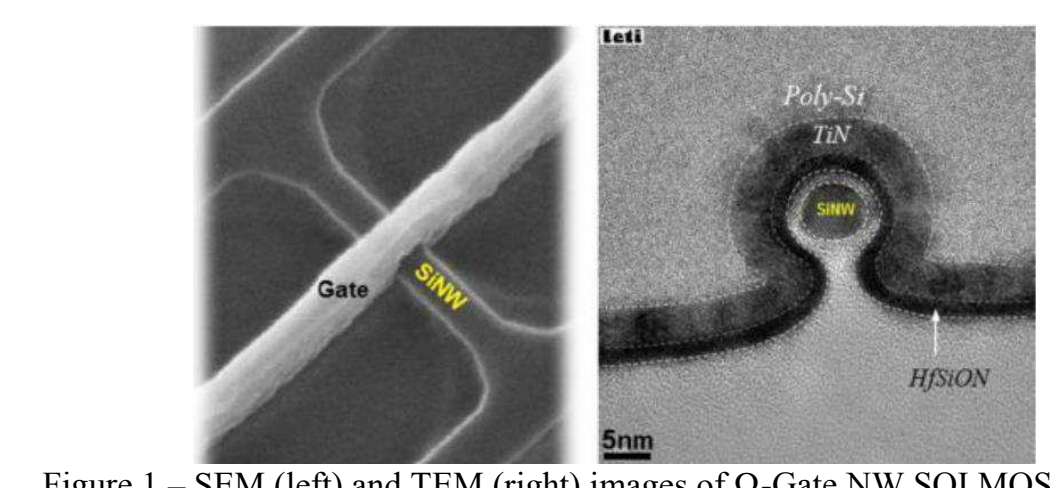

Figure 1 - SEM (left) and TEM (right) images of $\Omega$-Gate NW SOI MOSFETs (3).

The measurements of the devices were done varying the voltage from gate to source $\left(\mathrm{V}_{\mathrm{GS}}\right)$ from -2 to $1.2 \mathrm{~V}$ and with a drain to source voltage $\left(\mathrm{V}_{\mathrm{DS}}\right)$ of $50 \mathrm{mV}$.

\section{Results}

Figure 2 presents the curve of the drain current (IDS) as a function of gate voltage $\left(\mathrm{V}_{\mathrm{GS}}\right)$ from which the electrical parameters such as threshold voltage $\left(\mathrm{V}_{\mathrm{T}}\right)$ and subthreshold swing (SS) (Fig.3) were extracted. The curve for $\mathrm{W}_{\mathrm{NW}}=220 \mathrm{~nm}$ presents a displacement when compared with the other two curves. To understand the reason of this displacement, some simulations were done and they are presented in figure 4.

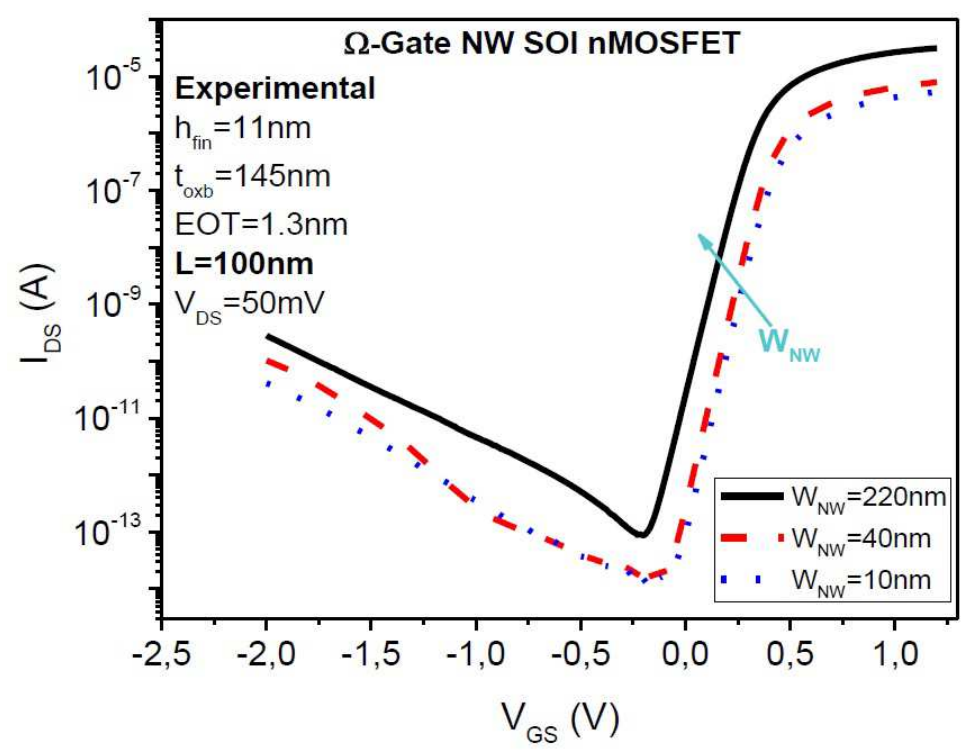

Figure 2 - Experimental drain current as a function of gate voltage, for channel length of $100 \mathrm{~nm}$ and $\mathrm{V}_{\mathrm{DS}}=50 \mathrm{mV}$.

Figure 3 presents the experimental extract threshold voltage $\left(\mathrm{V}_{\mathrm{T}}\right)$ and subthreshold swing (SS) of the three analyzed $\mathrm{W}_{\mathrm{NW}}$. It is possible to see that short channel devices present a SS degradation as well as present a $V_{T}$ variation for all channel widths, improving for narrow width as expected. However, focusing on $\mathrm{V}_{\mathrm{T}}$ behavior, an unexpected reduction on the $\mathrm{V}_{\mathrm{T}}$ values is observed for wider channel width even for long devices.

Aiming to better understand this unexpected behavior, some simulations were performed using TCAD Sentaurus, Synopsis ${ }^{\circledR}(4)$. It was simulated the device with channel 
width ( $\mathrm{W}_{\mathrm{NW}}$ ) of $220 \mathrm{~nm}$ and channel length (L) of $100 \mathrm{~nm}$, the others characteristics as the channel height $\left(\mathrm{h}_{\text {fin }}\right)$ were the same of the experimental devices.

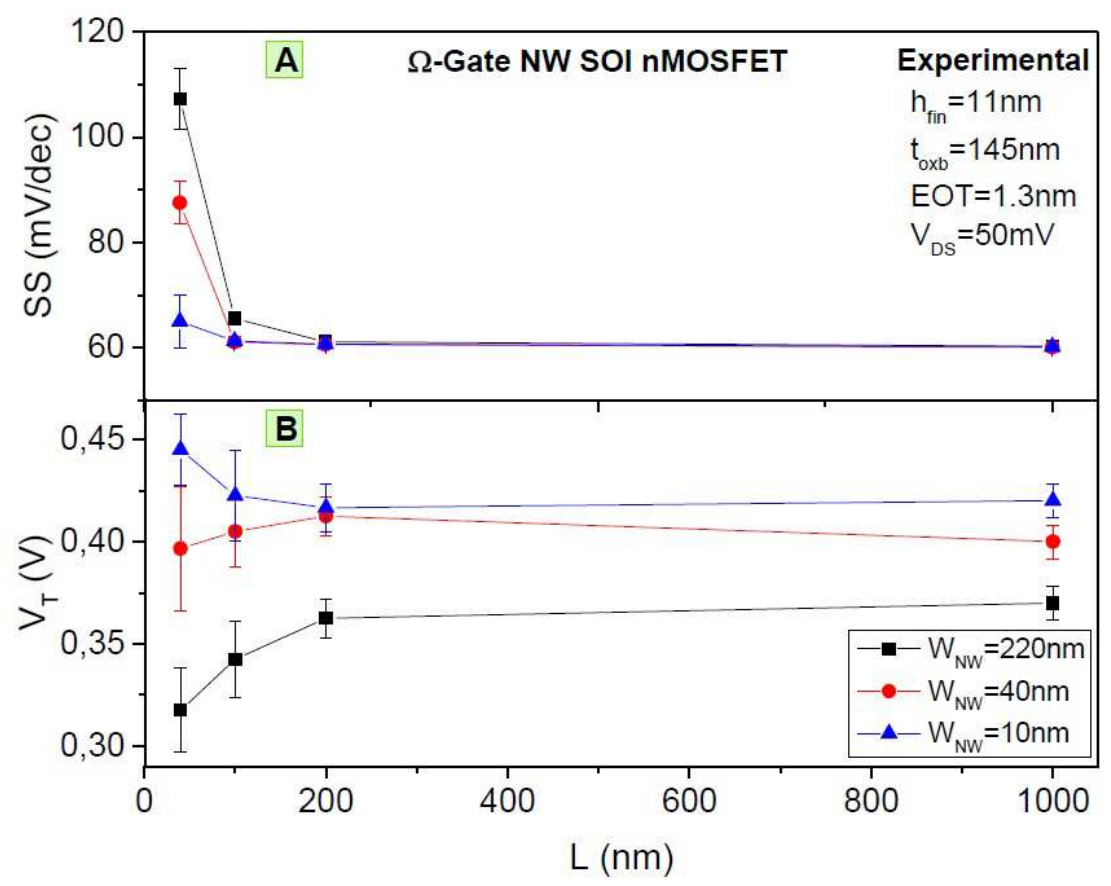

Figure 3 - Experimental subthreshold swing (A) and threshold voltage (B) as a function of channel length, for $\mathrm{V}_{\mathrm{DS}}=50 \mathrm{mV}$.

Figure 4, shows the simulated transfer characteristic for device with the same characteristics of the experimental one. It was observed that in order to have a good agreement of the simulated with the experimental data, it was necessary to include the interface fixed charges of $5 \times 10^{11} \mathrm{~cm}^{-3}$.

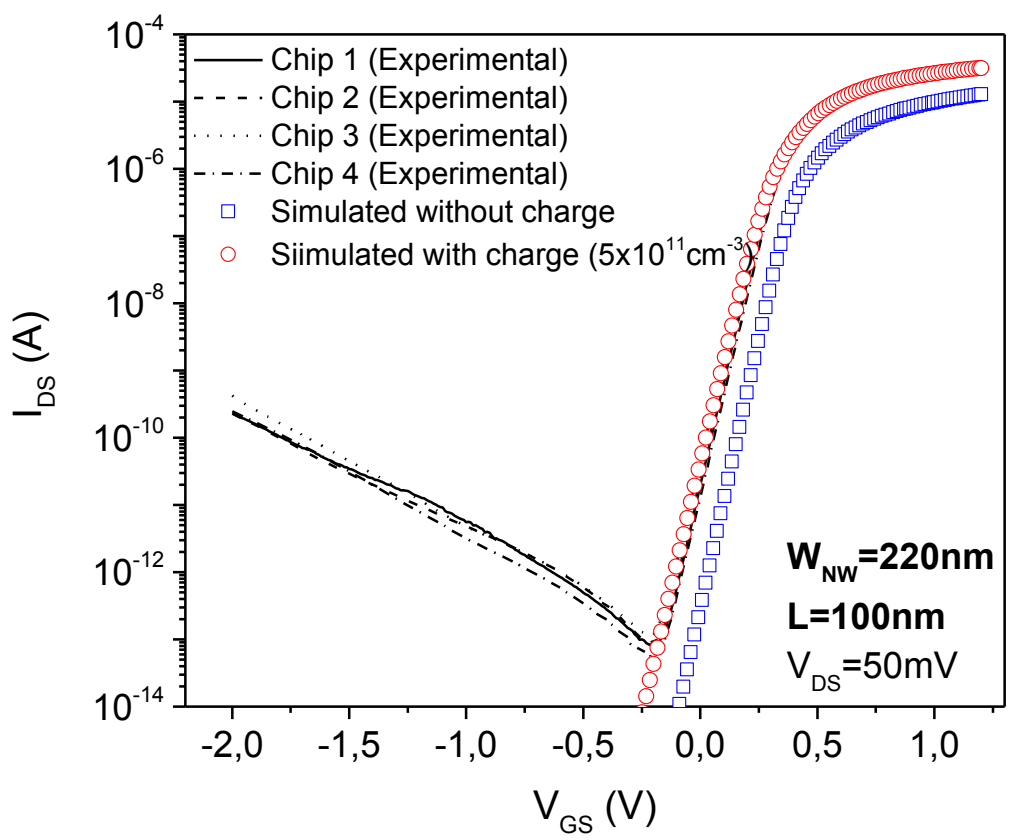

Figure 4 - Simulated drain current as a function of gate voltage for $\mathrm{W}_{\mathrm{NW}}=220 \mathrm{~nm}$ and $\mathrm{L}=100 \mathrm{~nm}$. 
The presence of positive fixed charges changes the back interface conduction condition, that means, the device can be conducting by the back interface (channel/buried oxide). To analyze this conduction, were done some simulations varying the fixed charge concentration from $5 \times 10^{11} \mathrm{~cm}^{-3}$ up to $1 \times 10^{13} \mathrm{~cm}^{-3}$ at back interface.

Figure 5 shows the curves with different fixed charges concentrations, where it is possible to notice that the increase of fixed charges causes a parasitic conduction from drain to source at the back interface.
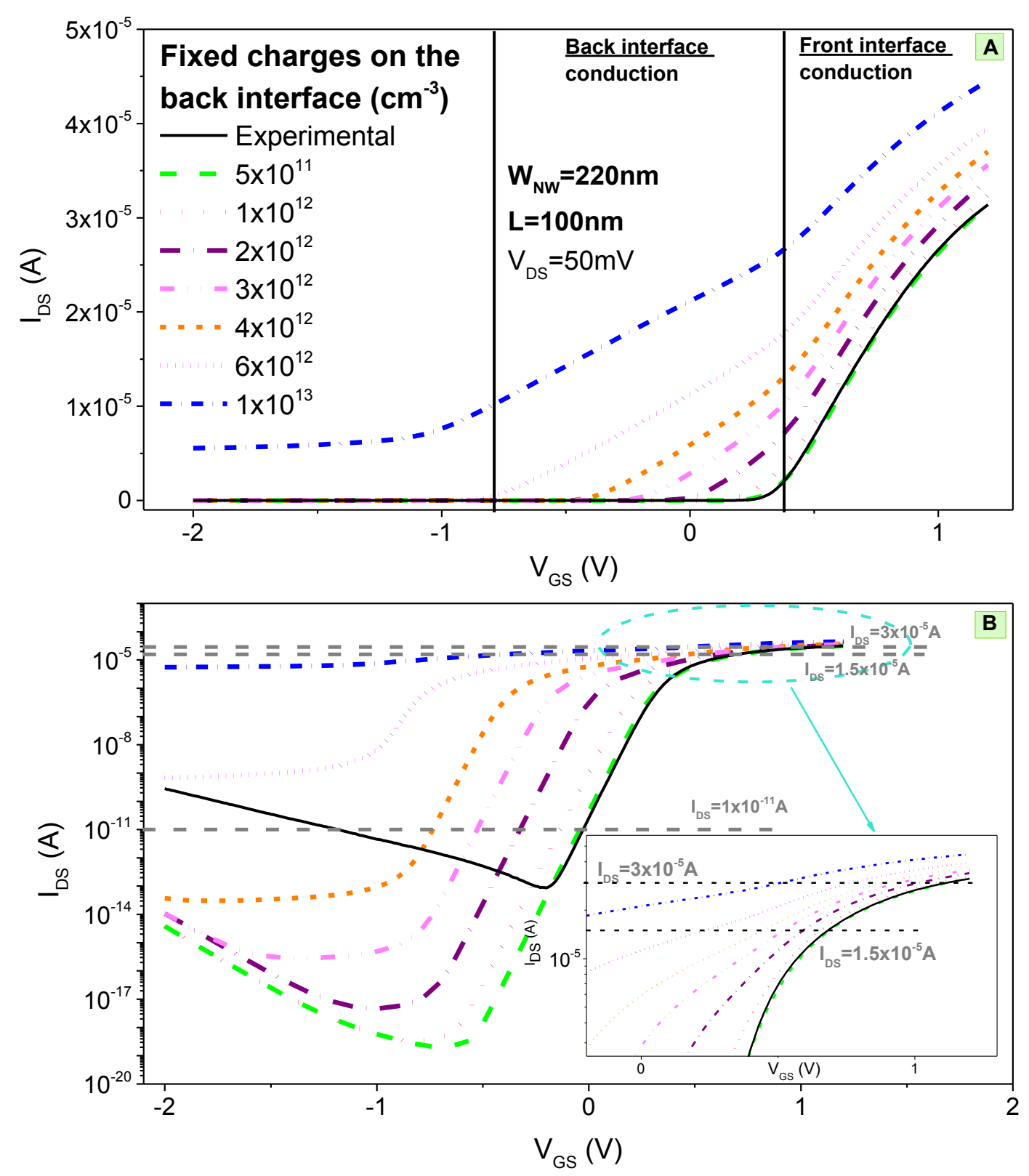

Figure 5 - Simulated drain current as a function of gate voltage in linear (A) and logarithmic (B) scale.

Aiming to analyze the back conduction, three different values of IDs were adopted, as can be seen in figure 5B. Analyzing the current density on the front and back interface in figure 6 , it can be noted that on the first point, that is located on the subthreshold slope (Fig.6A), the conduction is mainly composed by the back interface for all fixed charges level. When getting closer to $\mathrm{V}_{\mathrm{T}}$ (Fig.6B) the conduction by the front interface starts to increase but the device characteristics is still affect by the back conduction and then after 
$\mathrm{V}_{\mathrm{T}}$ (Fig.6C) the front interface predominates. The transition of predominant conduction from the back to the front interface usually causes an abnormal IDs behavior that can be seen in the inset presented in figure 5B, where a kind of kink is clearly observed in the curves which the fixed charges is higher than $3 \times 10^{12} \mathrm{~cm}^{-3}$.
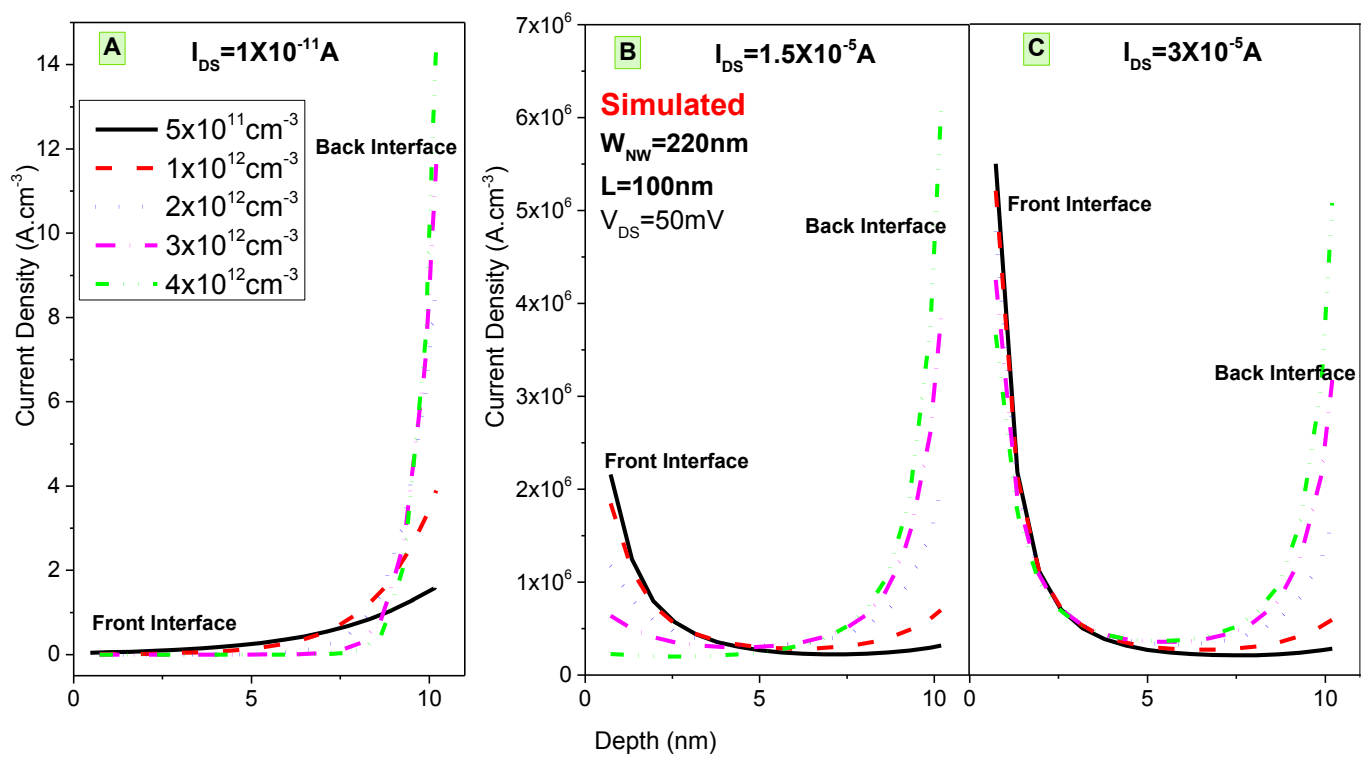

Figure 6 - Current density as a function of depth for three values of drain current.

Since the EOT is very thin, and the $\mathrm{h}_{\mathrm{fin}}$ is $11 \mathrm{~nm}$, for fixed charges smaller than $3 \times 10^{12} \mathrm{~cm}^{-3}$ the usual behavior degradation caused by back conduction (the SS increase or a hump in the subthreshold region) is not observed in the simulated results. This unexpected result can be explained by calculating the theoretical values of SS for the two different situations: 1) front and 2) back interface conduction, presenting and insignificant variation between the front and back SS values. This behavior can also be observed from the extracted SS values from the simulations varying the fixed charges, presented in table I. This SS immunity to back conduction happens because these devices have a very good coupling between the interfaces due to the thinner EOT and $\mathrm{h}_{\text {fin. }}$.

TABLE I - Subthreshold swing values for different fixed charges concentrations.

\begin{tabular}{cc}
\hline Fixed Charges $\left(\mathbf{c m}^{-3}\right)$ & SS (mV/dec) \\
\hline $5 \times 10^{11}$ & 65.1 \\
$1 \times 10^{12}$ & 65.7 \\
$2 \times 10^{12}$ & 66.1 \\
$3 \times 10^{12}$ & 68.1 \\
$4 \times 10^{12}$ & 68.2 \\
\hline
\end{tabular}

Although this device presents a higher SS immunity, the back interface conduction occurs early as the channel width becomes wider, resulting in smaller $\mathrm{V}_{\mathrm{T}}$ for all channel lengths. This behavior is more pronounced as the fixed charge increases on the back interface. This behavior can also explain the reduction on $\mathrm{V}_{\mathrm{T}}$ values for wider devices presented on the experimental results (Fig.3).

As the interface fixed charges did not present a significant variation on the SS, was performed some simulations adding the interface traps in the front interface (gate oxide/channel). The interface fixed charges were kept in $5 \times 10^{11} \mathrm{~cm}^{-3}$ and the interface traps 
concentrations were $1 \times 10^{12}$ and $5 \times 10^{12} \mathrm{~cm}^{-3}$. Figure 7 presents the simulated curves with the respective simulations.

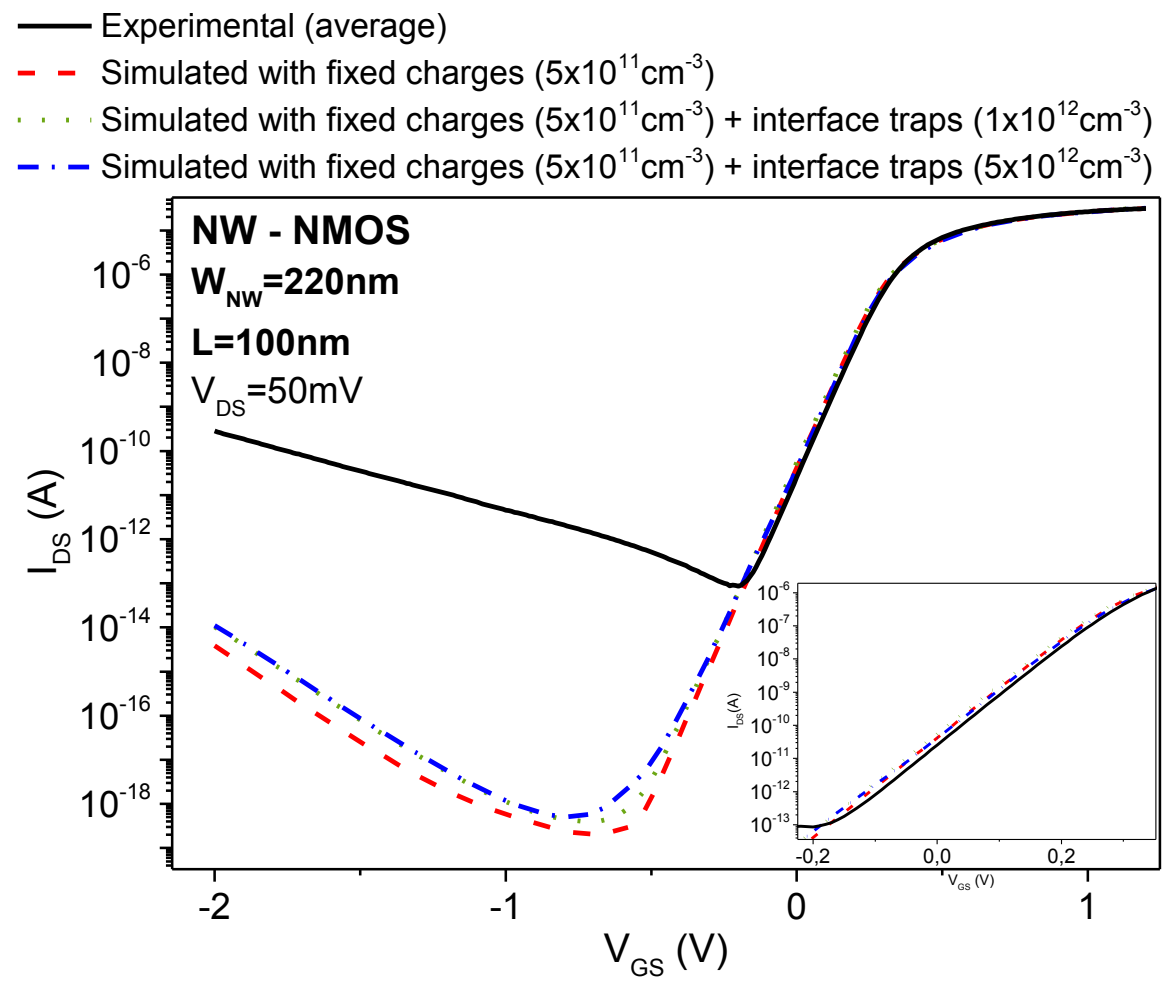

Figure 7 - Simulated drain current as a function of gate voltage with interface fixed charges and interface traps.

It is expected that the interface traps degrades the device performance, as a threshold voltage shift, subthreshold swing increase, and drain current degradation (5). However, when analyzing the simulations curves (Fig.7) and the SS extracted values from table II, it is presented an insignificant variation. Even when expanding the subthreshold region on the inset of figure 7 , it is impossible to note a SS variation.

This SS immunity to fixed charges and interface traps is related to the EOT and the silicon height $\left(\mathrm{h}_{\text {fin }}\right)$ of these devices that is very thin and provides a very good coupling between the interfaces and when adding interface traps it did not affect the device behavior.

TABLE II - Subthreshold swing values for different fixed charges and interface traps concentration.

\begin{tabular}{lc}
\hline Experimental (mean) & SS (mV/dec) \\
Fixed charges $\left(5 \times 10^{11} \mathrm{~cm}^{-3}\right)$ & 65.6 \\
Fixed charges $\left(5 \times 10^{11} \mathrm{~cm}^{-3}\right)+$ interface traps $\left(1.10^{12} \mathrm{~cm}^{-3}\right)$ & 65.1 \\
Fixed charges $\left(5 \times 10^{11} \mathrm{~cm}^{-3}\right)+$ interface traps $\left(5.10^{12} \mathrm{~cm}^{-3}\right)$ & 67.2 \\
\hline
\end{tabular}

\section{Conclusions}

The fixed charges on the back interfaces affects significantly the threshold voltage, reducing its values, due to the parasitic conduction on the back interface, reducing the control of $\mathrm{V}_{\mathrm{T}}$ by the voltage applied at the gate. This behavior was observed experimentally for $\mathrm{W}_{\mathrm{NW}}=220 \mathrm{~nm}$, and then confirmed by the numerical simulations.

The simulated fixed charges on the back interface and the interface traps did not presented a significant influence on the SS behavior for these devices. This immunity is 
provided due to the thinner gate oxide of $1.3 \mathrm{~nm}$ and the thinner silicon layer $\left(\mathrm{h}_{\text {fin }}\right)$ of $11 \mathrm{~nm}$ that provides a very strong coupling between the interfaces. Then, for $\mathrm{W}_{\mathrm{NW}}=220 \mathrm{~nm}$ even with a back interface conduction and that provides a reduction of the channel charges control by the gate voltage, there is no important degradation in SS.

\section{Acknowledgment}

The authors would like to thank FAPESP and CNPq for the financial support and the CEA-Leti for providing the samples for this work.

\section{References}

1. H. Soeleman and K. Roy, International Symposium on Low Power Electronics and Design, p. 94 (1999).

2. S. M. Sharroush, Ain Shams Engineering Journal, p. 1 (2016).

3. S. Barraud, R.Coquand, M.Casse, M.Koyama, J.M. Hartmann, V. Maffini-Alvaro, C. Comboroure, C. Vizioz, F. Aussenac, O. Faynot, T. Poiroux, IEEE Electron Device Lett., 33, p.1526 (2012).

4. Synopsys, Synopsys, Inc, $\mathbf{3}$ http://www.synopsys.com/Company/Pages/Trademarks. aspx. (2013)

5. T. K. Chiang, IEEE Trans. Electron Devices, 62, p. 2745 (2015). 